\title{
Extensive lichenoid sarcoidosis intermingled with white papules
}

Journal: The Journal of Dermatology

Manuscript ID: JDE-2009-0229.R2

Wiley - Manuscript type: Letter to the Editor

Keywords: sarcoidosis, tranilast, lichenoid, granuloma annulare, uveitis

\begin{abstract}
:
Cutaneous sarcoidosis is classified into nodular, plaque, diffuse infiltrative, and lichenoid types and others. Herein, we report an extensive case of rare lichenoid sarcoidosis with unusual small white macules representing early lesions of its typical red papules.
\end{abstract}




\section{Dear Editor,}

Cutaneous sarcoidosis is classified into nodular, plaque, diffuse infiltrative, and lichenoid types and others 1,2. Herein, we report an extensive case of lichenoid sarcoidosis with unusual small white macules representing early lesions of its typical red papules.

A 76-year-old Japanese man presented with mildly pruritic red papules on his back that had been there for 2 years. The eruptions were refractory to topical application of a very strong class of corticosteroid ointment. Physical examination showed that papules were reddish, round, flat, and up to 5-mm in diameter (Fig.1 a, b). They predominantly distributed in follicular pattern. In addition, numerous small white, slightly indurated macules were found mainly on the upper back (Fig. 1a, b). The red papules gradually spread to almost whole the body except face, hands and feet (Fig. 1c, d). He complained of severe hair loss in area with follicular papules especially of the lower limbs (data not shown).

Skin biopsies were taken from a red papule and a white macule. Analysis of a red papule revealed that numerous nodular granulomas throughout the entire dermis (Fig. 2a) as well as granulomas consisted of thin layers of epitheloid cells between collagen bundles presenting in an interstitial granuloma pattern (Fig. 2b). Those epithelioid cells were positive for CD68 (data not shown). In the superficial dermis, perivascular lymphocytic infiltration was observed (Fig 2a, inset). That of a white macule demonstrated fewer but discrete sarcoidal granulomas with giant cells in the mid to deep dermis (Fig. 2c, d), but no interstitial granuloma or no perivascular inflammatory infiltration in the superficial dermis. The presence of arrector pili muscle (Fig. 2c) suggested the lesion was close to hair follicle.

No bacteria or fungi were detected upon culture, Ziel-Nielsen and Grocott staining. PCR did not detect M. tubercle bacilli complex, M. avium, and M. intracellulare. QuantiFERON-TB Gold test was negative. All routine laboratory results were normal, including blood sugar, hemoglobin A1c, angiotensin-converting enzyme, serum calcium, and $\gamma$-globulin levels. Ophthalmologic examinations, chest radiography, chest computed tomography, gallium scintigraphy, and electrocardiography did not detect any abnormalities. The patient had been taking a calcium channel blocker and low-dose aspirin for hypertension, but his general health was otherwise good. Since no granulomatous lesions were detected in other organs, we tentatively diagnosed this case as an atypical case of generalized granuloma annulare (GA). We started to treat the patient with tranilast (N-3, 4-dimethoxycinnamoyl antheranilic acid) $300 \mathrm{mg} /$ day. Tranilast is an anti-allergic drug that has been used to treat atopic 
dermatitis or keloids in Japanese patients since the 1980s. While the precise underlying mechanism is unknown, it has been shown tranilast inhibits in vitro multinucleated giant cells formation through inhibiting the expression of adhesion molecule and purinergic receptor3 and that tranilast is also effective for other granulomatous disorders 4 . He noticed ocular defect at 8 months after our first evaluation, and eye examinations at this time revealed bilateral uveitis. Taken these into accounts, we diagnosed our case as lichenoid sarcoidosis. The eruptions started to subside at 6 months and almost disappeared at 10 months after our first evaluation. However, the eruptions repeated remission and exacerbation during the treatment of oral tranilast over half a year, and the efficacy of tranilast was not certain.

Differential diagnoses included sarcoidosis, interstitial granulomatous drug reaction (IGDR), generalized GA once when infectious granuloma was excluded. IGDR is a novel drug-associated entity5, and it was excluded because the eruptions continued to spread even after stopping medication. GA appears clinically in four different forms known as the localized, generalized, perforating, and subcutaneous forms, and four different patterns of infiltration can be observed, namely, palisading, interstitial, sarcoidal, and mixed6,7. Furthermore, because mucin deposition was found in the granuloma8, generalized GA with mixed histopathological features of interstitial and sarcoidal granuloma had not been denied until uveitis developed. A similar case of lichenoid sarcoidosis presenting follicular distribution all over the surface of the body was previously reported 9 , in which skin symptoms regressed spontaneously and also preceded the appearance of uveitis as our case.

The color of the granulomas lesions is theoretically thought to be skin color to white as seen in the cases of subcutaneous sarcoidosis unless there is inflammation in the upper dermis. Such inflammation often accompanies dilatation of blood vessels and lead to red color of the lesion. The white color of the early lesions of the lichenoid sarcoidosis as seen in this case may be explained by the presence of relatively deeper granuloma without inflammation in the upper dermis. It is also possible to postulate fine vessels in the upper dermis may be compressed due to the underlying granulomas. The small white macules have not been described as clinical phenotypes of lichenoid sarcoidosis.

In a couple of months, the white macules diminished as the red papules increased and spread. We further speculate that this case presented solid sarcoidal granuloma with giant cells in the early stage and changed to interstitial granulomas and developed the latter with inflammatory cells in the late stage.

The white macules were detected only in the early stage of this case. Thus, we should be 
careful not to overlook such early lesions of lichenoid sarcoidosis.

\section{References}

1 Newman LS, Rose CS, Maier LA. Sarcoidosis. N Engl J Med 1997; 336:

1224-34.

2 English JC, 3rd, Patel PJ, Greer KE. Sarcoidosis. J Am Acad Dermatol 2001; 44: 725-43; quiz 44-6.

3 Okamoto H, Mizuno K, Horio T. Monocyte-derived multinucleated giant cells and sarcoidosis. J Dermatol Sci 2003; 31: 119-28.

4 Araki E, Kambe N, Takahashi K et al. Multiple dermatomal daughter lesions of postzoster granuloma. Br J Dermatol 2007; 156: 1369-71.

5 Magro CM, Crowson AN, Schapiro BL. The interstitial granulomatous drug reaction: a distinctive clinical and pathological entity. J Cutan Pathol 1998; 25:

$72-8$.

6 Friedman-Birnbaum R, Weltfriend S, Munichor M et al. A comparative histopathologic study of generalized and localized granuloma annulare. Am J Dermatopathol 1989; 11: 144-8.

7 Lynch JM, Barrett TL. Collagenolytic (necrobiotic) granulomas: part 1--the "blue" granulomas. J Cutan Pathol 2004; 31: 353-61.

8 Dabski K, Winkelmann RK. Generalized granuloma annulare: histopathology and immunopathology. Systematic review of 100 cases and comparison with localized granuloma annulare. J Am Acad Dermatol 1989; 20: 28-39.

9 Fujii K, Okamoto H, Onuki M et al. Recurrent follicular and lichenoid papules of sarcoidosis. Eur J Dermatol 2000; 10: 303-5. 


\section{Figure legends}

Figure 1.

Hundreds of small white macules and a few red papules on the upper back (a) and its close-up view (b) at our first examination. Arrows heads indicate representative white macules. The majority of red papules arose from white macules and were follicular papules. Three months (c) and 6 months (d) after our first examination.
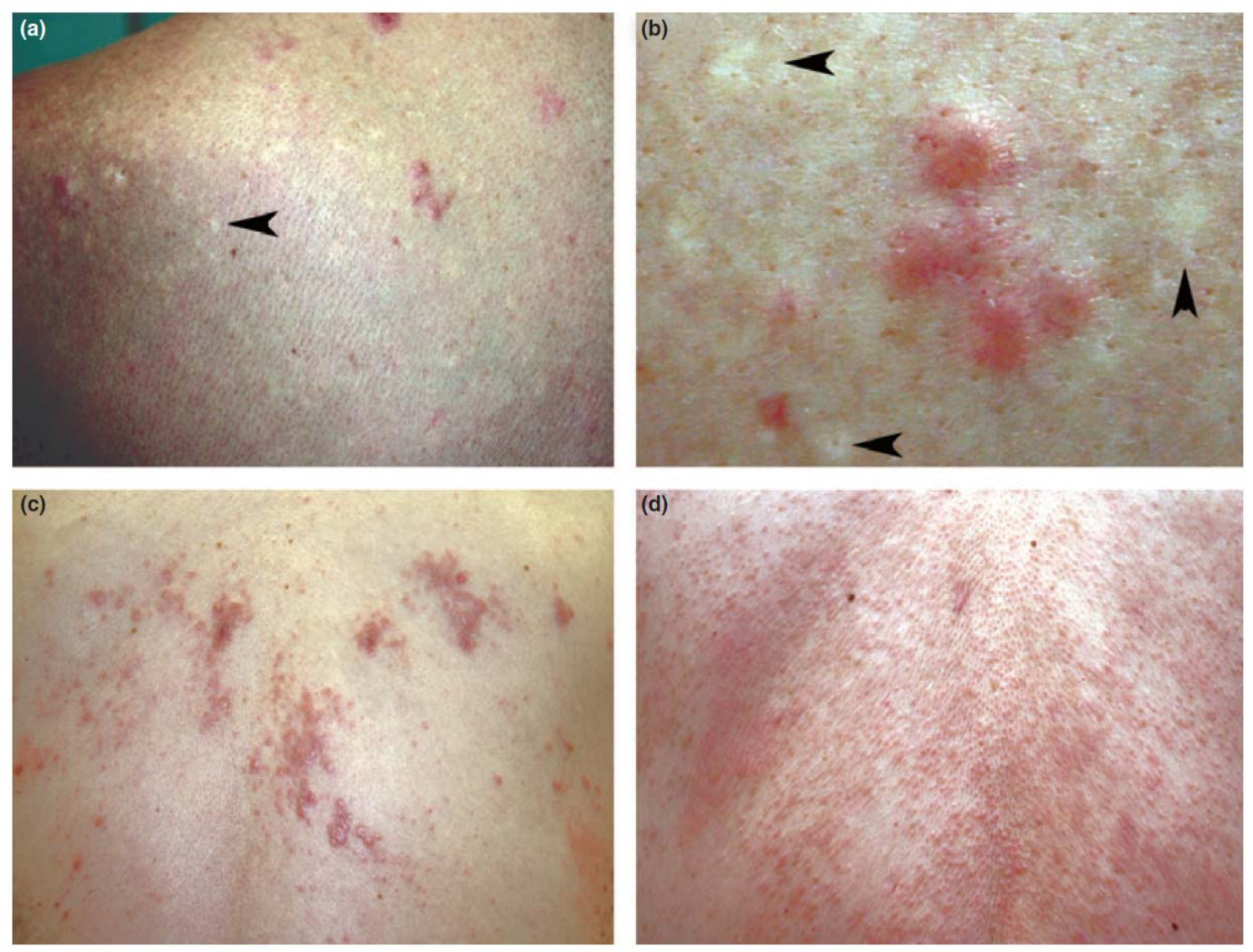
Figure 2.

(a-c) Histopathology of a red papule revealed that numerous nodular granulomas throughout the entire dermis (a) and interstitial granulomas consisted of thin layers of epitheloid cells between collagen bundles (b). That of a white macule revealed fewer but discrete granulomas in the mid to deep dermis (c, d). (Hematoxylin and eosin staining; a, c, x40; b, d, x400). The inset of (a) is a magnified picture of the superficial dermis showing perivascular infiltrate of lymphocytes. Arrow in (c) depicts arrector pili muscle.
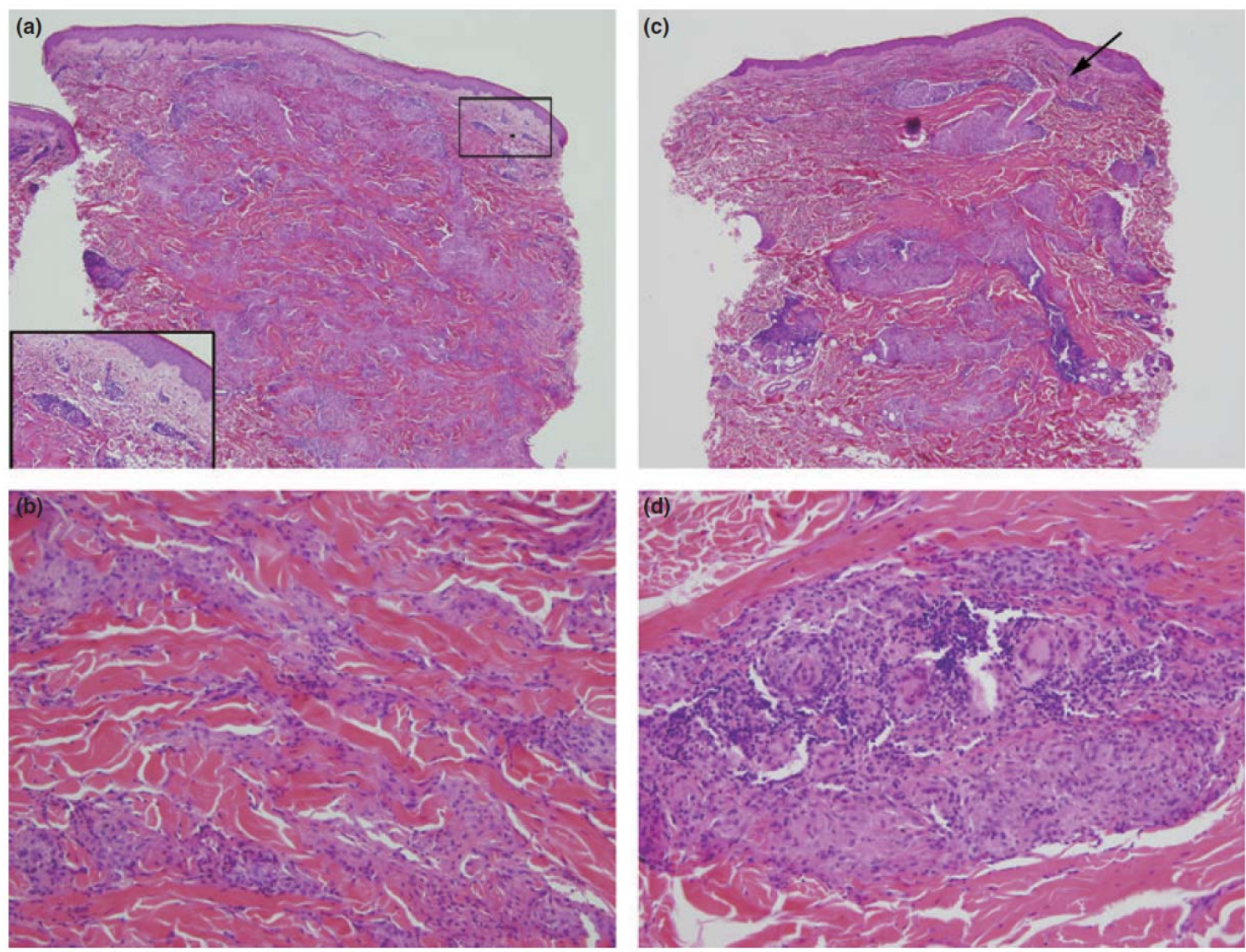